\title{
Genome-wide cell-free DNA methylation profiling in lung cancer patients
}

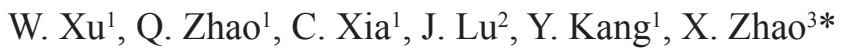 \\ ${ }^{1}$ Bio-ID Center, School of Biomedical Engineering, Shanghai Jiao Tong University, Shanghai, China. \\ ${ }^{2}$ Department of Pulmonary Medicine, Shanghai Chest Hospital, Shanghai Jiao Tong University, \\ Shanghai, China \\ ${ }^{3}$ Shanghai Center for Systems Biomedicine, Shanghai Jiao Tong University, Shanghai, China. \\ *E-mail: xiaodongzhao@sjtu.edu.cn
}

Key words: cfDNA, DNA methylation, MeDIP, next generation sequencing, non-small cell lung cancer

Motivation and Aim: As a non-invasive blood testing, the detection of cell-free DNA (cfDNA) methylation in plasma is raising increasing interest due to its diagnostic and biology applications [1]. Although extensively used in cfDNA methylation analysis, bisulfite sequencing is less cost-effective. Through methylated DNA immunoprecipitation coupled with deep sequencing (MeDIP-seq), we aimed to characterize cfDNA methylome in cancer patients.

Methods and Algorithms: In this study, we investigated the cfDNA methylation patterns in lung cancer patients by MeDIP-seq. MEDIPS package was used for the identification of differentially methylated regions (DMRs) between patients and normal ones.

Results: Overall, we identified 128 differentially methylated regions (DMRs) in gene promoter regions, 21 hypermethylation and 107 hypomethylation respectively, by comparing lung cancer patiens and healthy individuals as controls. 21 hypermethylation regions represent 20 genes. Some of the genes had been previously reported to be associated with lung cancers, such as CPXM1 and C1orf210.

Conclusion: Taken together, our study provided an alternative method of cfDNA methylation analysis in lung cancer patients with potential clinical applications.

Acknowledgements: This work was supported by Development Program for Basic Research of China (2014YQ09070904), National Natural Science Foundation of China (31671299), Shanghai Science and Technology Committee Program (17JC1400804), Medical engineering cross fund (YG2017ZD15, YG2015QN35) and Laboratory Innovative Research Programme of Shanghai Jiao Tong University (17SJ-18).

\section{References}

1. Feng H., Jin P., Wu H. (2018). Disease prediction by cell-free DNA methylation. Briefings in bioinformatics. 